\title{
A Frame work for improvising Service of Multimedia Applications in Mobile Networks
}

\author{
Bhuvaneswari Mariappan ${ }^{1}$ and Seethalakshmi Pandian ${ }^{2}$ \\ ${ }^{1}$ Department of Electronics and Communication Engineering, Anna University of \\ Technology, Tiruchirappalli \\ Tiruchirappalli-620024,Tamilnadu, India \\ bhuvanakirranegmail.com \\ 2 Centre for Academic Excellence, Anna University of Technology, Tiruchirappalli \\ Tiruchirappalli-620024, Tamilnadu, India
}

\begin{abstract}
The main objective of this paper is to propose a frame work for improvising the service of multimedia applications in mobile networks. The framework has been proposed with a Cross-Layer Manager (CLM) and a Cross-Layer Coordinator (CLC) for improvising of multimedia applications by dynamically adapting the bandwidth under prevailing channel conditions. Cross-Layer coordination among Application, MAC and Physical layer has been achieved along with bandwidth adaptation that improvises the Quality of Experience $(Q \circ E)$ of multimedia applications. The performance of the framework has been tested using GloMoSim and the results for the proposed framework under different scenarios show an improvement of $16 \%$ in throughput. It is also observed that our framework provides $5 \%$ improvement in multimedia service when compared with the cellular system containing only homogeneous traffic calls.
\end{abstract}

\section{KEYWORDS}

Quality of Service (QoS), Quality of Experience (QoE), Mobile Multimedia Networks, Cross-Layer Manager (CLM), Cross-Layer Coordinator (CLC), Bandwidth Adaptation Algorithm.

\section{INTRODUCTION}

The demand for multimedia applications in mobile networks is growing day by day. Recently mobile network service providers are concentrating more on provisioning of multimedia services such as online video chatting, mobile classroom educational system, mobile entertainment and mobile facebook in addition to its conventional voice services. Providing such multimedia services to the end users with the expected Quality of Experience (QoE) and Quality of Service (QoS) within the available bandwidth is a challenging task. QoS is a broad term used to describe the Quality of Experience (QoE) a user will receive over a network. In order to meet the demand of mobile end users, a framework with bandwidth adaptation for enhancing the QoS and QoE of multimedia applications using Cross-Layer coordination among Application, MAC and Physical layer is proposed.

A recent study in [1] has indicated that, traditional layered OSI model may not be well suited for high-speed bursty traffic in cellular networks. In cellular networks, the request from the mobile station (MS) is forwarded to the base station for channel allocation. The base station receiver should respond to the arrival of each such packet from mobile station (MS) in a very timely manner as, the packets from different mobiles carry completely different channel characteristics.

DOI : 10.5121/ijma.2011.3206 
Then, the base station receiver should execute all necessary algorithms such as channel estimation along with interactions with all upper layers within a very short time to make the detection of each packet successfully. But, in traditional OSI model due to adjacent layer interaction and the slow parameter exchanges across different layers, the base station receiver cannot respond instantaneously to the mobile station. So, traditional OSI model is not suitable for cellular networks as it may not work properly for high speed bursty traffic causing excessive latency in parameter exchanges across different layers. Hence, the cross-layer network design can help to reduce the overall response time at the transceiver by interaction between different layers through other interfaces for achieving a particular quality of service (QoS). So,CrossLayer Design (CLD) is a cooperative communication between the layers to increase the overall network capacity with better Quality of Service (QoS) for multimedia users. In the proposed framework, to achieve such a cooperative communication between layers some adapting algorithms using an evolutionary approach between Application layer, MAC layer and Physical layer to provide the required QoE with QoS to the requesting end users has been proposed.

In the proposed system, the module Cross-Layer Manager (CLM) in Base Station Switching Centre (BSC) generates and forwards control probe request signal for all requested calls to Mobile Switching Centre(MSC). The control probe request signal triggers the Cross-Layer Coordinator (CLC) of MSC for bandwidth adaptation to serve the request. In bandwidth adaptation, the available bandwidth is shared efficiently among the requesting calls by crosslayer interaction among Application, MAC and Physical layer.

In cross-layer interaction, the information regarding the traffic type of the requested call from the Application layer interacts with the MAC layer to probe whether required channel is available under prevailing channel conditions. If channel is available, MAC layer probes the Physical layer to send the Channel State Information (CSI) of the destined user.CSI message contains the value of required transmission power in every frame for each user with status of the channel. MSC then responds through ACK signal to BSC for channel allocation to requesting user. If required channel is not available, then bandwidth adaptation algorithm is applied to serve the requested mobile user.

However, in the conventional system, the allocated bandwidth is shared among multiple requesting users and if no bandwidth is available for further requesting users, they are simply denied of service. To overcome this abrupt service denial, bandwidth adaptation with cross-layer interaction approach is carried to meet the required QoS and QoE to mobile users.

In bandwidth adaptation, as far as channel is available, maximum bandwidth is first allotted to voice calls, then acceptable QoS bandwidth is assigned to video calls and minimum bandwidth to background and interactive calls. After this first level assignment, the residual bandwidth is further distributed in stepwise to the calls based on their traffic class as bandwidth up gradation process until the maximum capacity of the cell is attained. In order not to deprive the basic voice service of cellular networks, priority is always assigned to voice calls and then to video calls, then to background and interactive calls.

In bandwidth degradation, the allocated bandwidth of background and interactive calls is first degraded to minimum level to serve the requesting voice and video users under scarce bandwidth condition. After first level degradation, if still bandwidth is required for the requesting voice and video calls, then as a second level of bandwidth degradation, the allotted maximum bandwidth of video calls are degraded to acceptable QoS bandwidth level without disturbing the mobile multimedia users. 
The International Journal of Multimedia \& Its Applications (IJMA) Vol.3, No.2, May 2011

The paper is organized as section 2 with related works, section 3 with proposed methodology, bandwidth adaptation procedure in section 4, results and discussion in section 5 followed by conclusion in section 6 and references .

\section{RELATED WORKS}

The popular research work in QoS identified from literature [7]are European Research Funded Projects, Two IST STREP and 4 MORE-MC-CDMA Multiple- Antenna System On Chip for Radio Enhancements. Its main objective is to emphasize on research, develop, integrate and validate a cost effective low power system on chip solution for multi antenna multi carrier CDMA Mobile terminals based on joint optimization of layer 1 and layer 2.To develop an end to end optimized wireless communication link C.Verikoukis et al [7] proposed PHOENIX, a scheme offering the possibility to let the application world (source coding, ciphering) and the transmission world (channel coding, Modulation) talk to each other over an IPV6 protocol stack. [7] also proposed NEWCOM, IST-507325, NEWCOM Project E of IST Network of Excellence on Wireless Communication proposal to identify existing gaps in European Knowledge in crosslayer and also investigated the potential benefits of cross-layer in wireless network design in relation to the methodology of separate layer design. It also considered the coupling of higher layers with physical layer and elaborated the information to be exploited from the physical layer to optimize the network performance.

Cross-Layer Issues for 4G Networks was proposed by Gustavo Carnerio et al [8] as ERACLIN -Enhanced Radio Resource Algorithms based on Marie-Curie transfer knowledge project. They analyzed different cross-layer techniques to enhance the efficiency of wireless communication systems. A comprehensive study on the potential benefits that could be obtained from using these techniques in different environments and in particular the explicit shift toward decentralized and adaptive MAC, radio resource management and routing approaches were also carried out.

However, overview of problems faced by all IP wireless mobile terminals (often referred to as $4 \mathrm{G}$ terminals) was proposed in reference [8]. A simple framework for studying and solving these problems with Cross-Layer design has been presented. This framework first classifies known problems in four coordination planes - Security, QoS, Mobility and Wireless link. [8] Concluded that security problem arise from multiple-layer encryption, with unnecessary power consumption and processing delay. It also analyzed QoS problems affecting flows with QoS requirements caused by layer congestion control and link layer ARQ. The mobility problems are related to the effects of handover on transport layer connections with QoS signaling. Finally [8] concluded that wireless problems caused by packet corruption and losses that are perceived by TCP as congestion indications causes poor performance. In order to help solve these problems, a simple interlayer coordination model was presented in [8], consisting of a Cross-layer manager to receive event notifications from each protocol and perform management algorithms to control the internal state of each protocol in order to correct their behavior as per information from other layers. However, the author has concluded that future work will include the development of algorithms for management.

The Next Generation Mobile Networks (NGMN) design issues including architecture design, Cross-layer coordination, vertical hand-off and admission control in a multi traffic environment was addressed by Rubaiyat Kibria et al [9] . The proposal presented in this article provided valuable insight into the development of NGNM, but still a lot of work is necessary in areas of open research such as energy efficient multimode terminal development, session and personal mobility consideration with terminal mobility, dimensioning of both the radio and core network within the NGNM framework, Standardization of the inter working protocols and so forth. The 
ensuring proposal need to be designed in such a way that they reduce the amount of changes introduced into the existing systems and promote interoperability within the hierarchical framework.An overview of the cross-layer paradigm shift that is beginning to take place as wireless communication evolves from a circuit-switched infrastructure to a packet -based infrastructure was discussed by Bader Al-Manthari et al [10]. The advantages of Cross-layer networking offered through wireless LAN interface approach was also discussed in [10]. Based on observations, it appears that the above mentioned research areas are important for successful built out of future broadband wireless network. This paper provides viewpoint of the technical challenge and emerging research areas that lead to new network implementation to support the amalgamation of user services with QoS requirements.Further, energy efficient and low-power design within all layers of the wireless network protocol stack was addressed by Tianmin Ren et al [11] . The impact of higher order error statistics on the various layers of the protocol stack was also addressed. The author discussed the impact of such information for adaptive protocol design throughout the stack with power aware protocol in adhoc networks. References there in provided insight into the various power aware protocol proposals with design issues and a conceptual framework for network and client adaptation survey regarding the various proposals for application adaptation and map it to the conceptual framework. A survey on various suggestions to improve TCP behavior over heterogeneous network was also discussed.

However, some possible approaches of QoS Enhancement in Multimedia Mobile Networks identified from literature includes IMS [IP Multimedia subsystem] to support multimedia traffic with QoS along with Software Defined Radio (SDR) to provide access to network independent services [12] with IPV6 forming common platform for $4 \mathrm{G}$ networks. To achieve higher data rate, modification of radio and core network could be done to enable new as well as emerging networks for seamless connectivity to the general framework [13].Cross-Layer coordination among different layers is facilitated through well defined message interfaces such as Application Programming Interface( API), Inter Signaling Pipe (ISI) and ICMP. A common control/signaling mechanism could be utilized to assist access network discovery, location management and vertical handoff by periodically computing and broadcasting a list of available Radio Access Network (RANs). List of surrounding BS IDs, their associated AR IDs and network alternatives and their QoS parameters are analyzed in [9]. However, the common signaling problem could be resolved using an overlay structure on existing BS / APS (as proposed by MIRAI)[13].

For resource management, a distributed bandwidth broker (BB) is proposed inside each router domain, along with backup facility where BB includes SLA, SLS, ACS, A\&A, PDP [13]. Specifically Cross-layer coordination for Application and Network layer could only be provided by using separate external servers for time critical services. In $4 \mathrm{G}$ networks in order to provide connectivity at anytime along with horizontal hand-off, forced vertical hand-off was also proposed to upgrade the QoS of application against dropped sessions. To be specific, periodical vertical handoff algorithm could also be used. This algorithm can be embedded within terminal architecture for Mobile Terminal controlled against Mobile Terminal assisted handoffs as in $3 \mathrm{G}[14]$.

Problems pertaining to varying network conditions such as latency, bandwidth and energy could be rectified using watermark based mechanism along with ICMP messages in Cross-layer coordination as proposed in [7].As 4G deals with heterogonous networks, so for access network selection, the proposed method will be Analytic Hierarchy Process (AHP) and Grey Relational Analysis (GRA) taking throughput, delay, jitter, reliability, BER, burst error, average retransmissions, security, cost \& power consumption [15]. For QoS normalization, the proposed algorithm takes smaller the better concept (timeliness, reliability, cost and power consumption). Likewise larger the better would also be referred for throughput and security. 
To get fair Call Admission Control (CAC) algorithm in a multi traffic environment, adaptive Markov -Model based CAC policy was proposed. For testing advanced mobile services, based on NTP - SIOT [Service Interoperability Test] TTCN-3 [Taiwan] platform could be used as test benches for OMA [Open Mobile Alliance] multimedia messaging service exclusively for $4 \mathrm{G}[21]$.

\section{PROPOSED FRAMEWORK}

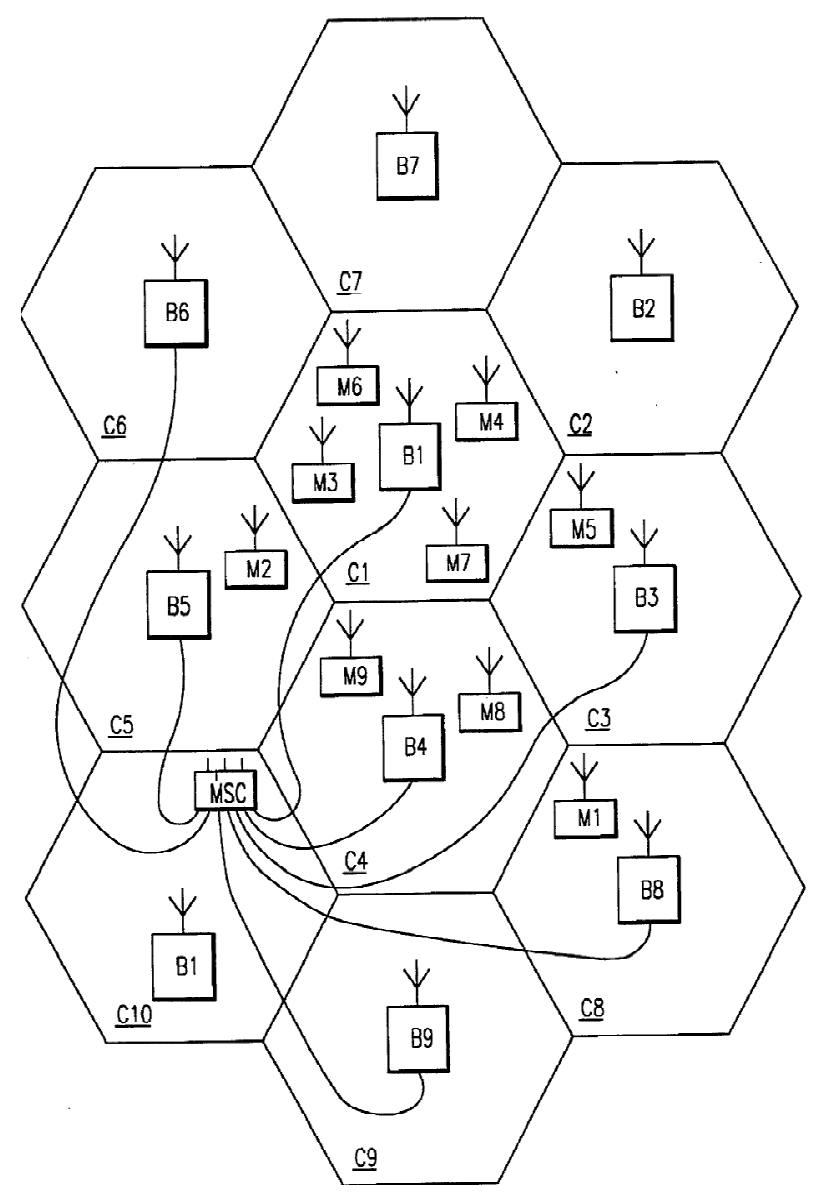

Fig (3.1) Architecture of Cellular Network

M-Mobile Station, B-Base Station, C-Cell site

In Cellular mobile networks, when a mobile user requests for a call connection, the request is forwarded to BSC.BSC then forwards this request to MSC regarding the allocation of channel to the requested mobile user.MSC in turn probes the availability of the destination and based on the availability it instructs the BSC either to allocate or deny the request. However, in the conventional system, the allocated bandwidth is shared among multiple requesting users and if no bandwidth is available for further requesting users, they are simply denied service. To overcome this abrupt service denial, in the proposed framework, bandwidth adaptation with cross-layer interaction between Application, MAC and Physical layer approach is carried out to meet the required QoS namely throughput with minimized delay for efficient network utilization to mobile users. 
The International Journal of Multimedia \& Its Applications (IJMA) Vol.3, No.2, May 2011
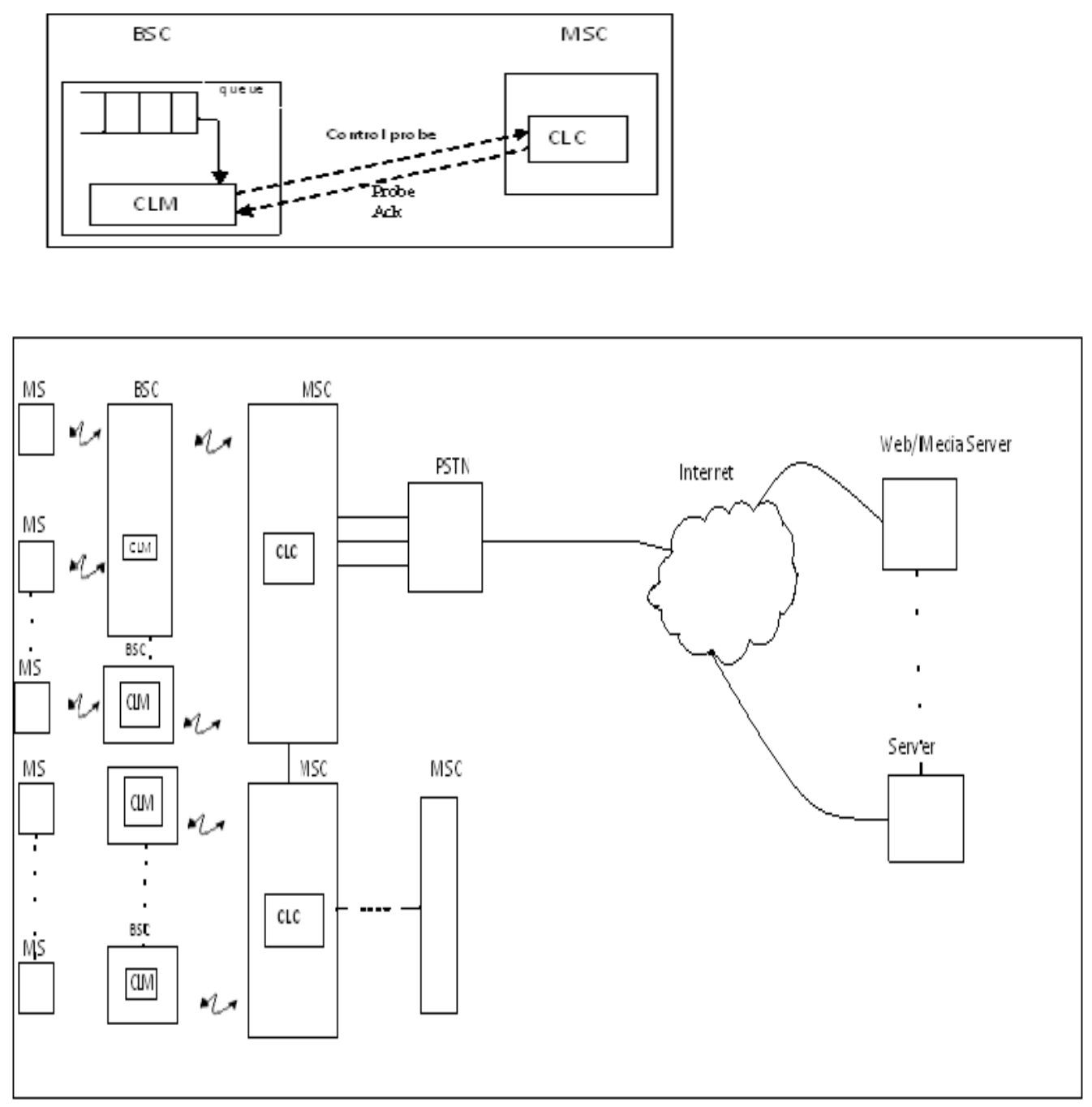

Fig (3.2) Proposed Framework model for Cellular Networks 
The International Journal of Multimedia \& Its Applications (IJMA) Vol.3, No.2, May 2011

Cross-Layer Manager- CLM

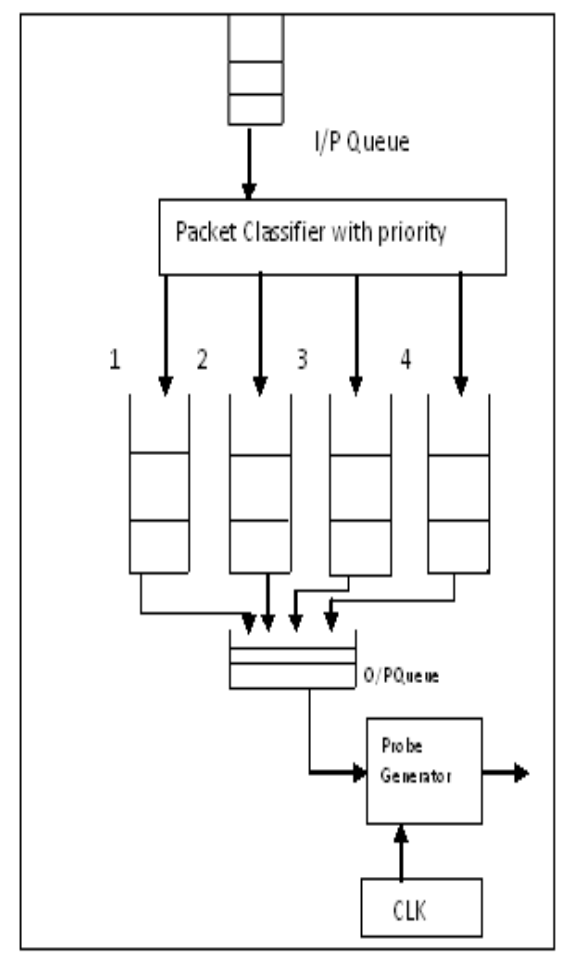

Cross-Layer Coordinator- CLC

$\mathrm{CLC}$

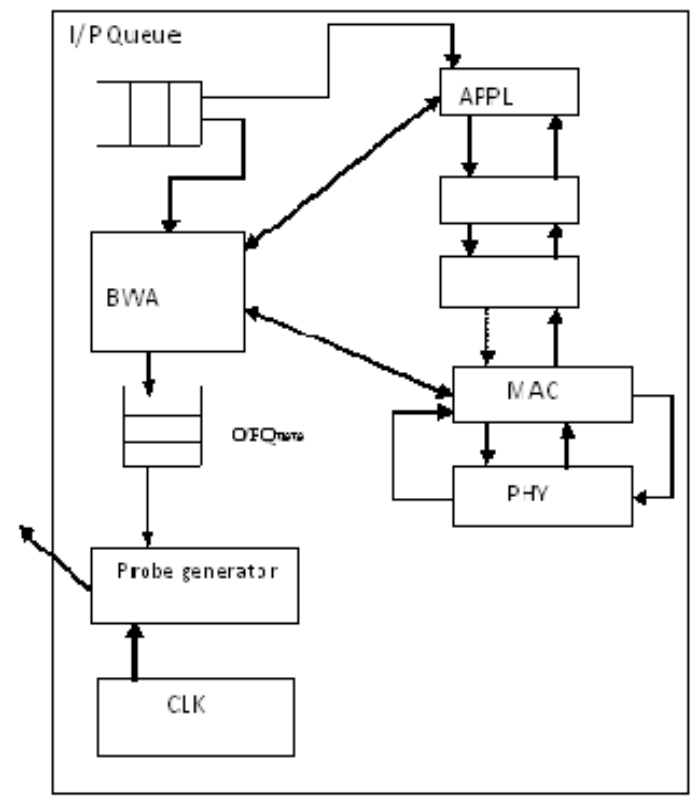

Fig (3.3) Internal Block Details of the Proposed Model

In the proposed framework, depending on the type of application, priority is assigned as per IEEE802.11e standard to achieve QoS. The proposed system consists of Cross-Layer Manager in BSC which generates control probe request signal for each requested calls in its queue. The requesting calls from users are queued as they arrive and classified based on their traffic in packet classifier. Four queues, namely Vo for voice, Vi for video, BE for best effort services and BK for background services are taken similar to IEEE 802.11e.As in [18] IEEE 802.11e in MAC provides QoS for multimedia such as video chatting traffic and other (VRT) virtual class room based real time traffic. Priority is assigned to Vo, then to Vi, then to best effort services and finally to background services in order not to deprive the basic voice services of cellular networks. The calls which are in the output queue are served one by one when a synchronized clock generates a probe signal at each tick. Thus, for each prioritized calls from output queue and at the tick of the clock, a probe request signal is generated by the probe generator. The control probe request signal triggers the Cross-Layer Coordinator of MSC.

The Cross-Layer Coordinator in turn triggers the Application layer with the information regarding the traffic type and the required channel of the requesting user. Application layer interacts with the MAC layer to probe whether the channel is available for the requested 
application. If channel is available, MAC layer probes the Physical layer to send the Channel State Information (CSI) of the destined user. CSI message contains the value of required transmission power in every frame for the user with channel status. MSC then instructs BSC to allocate the required number of channels to the requesting user for information exchange. If the required channel is not available, then MSC performs bandwidth adaptation procedure to serve the requesting call.

\section{BANDWIDTH ADAPTATION ALGORITHM}

The traffic classes namely voice, video/multimedia, best effort and background are represented as class-I, class -II, class -III and class-IV respectively. The traffic classes are assigned a minimum required bandwidth, an acceptable QoS bandwidth and maximum required bandwidth as given in table (1).

\begin{tabular}{|c|c|c|c|c|c|}
\hline S.No & Class & Traffic Type & $\begin{array}{l}\text { Minimum } \\
\text { required } \\
\text { bandwidth in } \\
\text { Mbps }\end{array}$ & $\begin{array}{l}\text { Acceptable } \\
\text { QoS level } \\
\text { bandwidth in } \\
\text { Mbps }\end{array}$ & $\begin{array}{l}\text { Maximum } \\
\text { level } \\
\text { bandwidth in } \\
\text { Mbps }\end{array}$ \\
\hline 1 & Class-I & voice call & $\mathrm{a} 1=0.03$ & ------ & $\mathrm{a} 1=0.03$ \\
\hline 2 & Class-II & $\begin{array}{l}\text { video and } \\
\text { multimedia } \\
\text { calls Vi }\end{array}$ & $\mathrm{b} 1=2$ & baccept $=4$ & $\mathrm{~b} 2=5$ \\
\hline 3 & Class-III & Best effort BE & $\mathrm{c} 1=1$ & ------ & $\mathrm{c} 2=2$ \\
\hline 4 & Class-IV & $\begin{array}{l}\text { Background } \\
\text { BK }\end{array}$ & $\mathrm{d} 1=0.003$ & ------ & $\mathrm{d} 2=0.02$ \\
\hline
\end{tabular}

The number of calls in each traffic class is represented by (nx,ny,nz,ne) respectively, where

$$
\begin{aligned}
& \mathrm{nx}=\mathrm{nx} 1, \mathrm{nx} 2,, \ldots \ldots \ldots \ldots . . \mathrm{nxn} \quad \text { for class }-\mathrm{I} \\
& \text { ny }=\text { ny } 1, \text { ny } 2, \ldots \ldots \ldots . . . . . n y n \quad \text { for class }- \text { II } \\
& \mathrm{nz}=\mathrm{nz} 1, \mathrm{nz} 2, \ldots \ldots \ldots \ldots \ldots . \mathrm{nzn} \quad \text { for class }- \text { III } \\
& \text { ne }=\text { ne } 1, \text { ne } 2, \ldots \ldots \ldots . . . . . . n e n \quad \text { for class }-\mathrm{IV} \\
& \text { C -Total capacity of the system }
\end{aligned}
$$

Let ai, bj, ck, de represents the bandwidth requirement of class-I, class-II, class-III and class-IV traffic respectively and i,j,k,e represents the levels of required bandwidth by the application.

The state space for the system is

$$
\text { ainx }+ \text { bjny }+ \text { cknz }+ \text { dlne }<\text { c } \quad \rightarrow(4.1)
$$

Equation (4.1) should not be violated during allocation of bandwidth to each traffic class.

Initially assume there are 3calls in class -I, 2calls in class-II, 1 call in class-III and 1 call in class IV. This could be represented as $(3,2,1,1)$. The state space equation for $(3,2,1,1)$ is

$$
\begin{gathered}
\text { ainx } 1+\operatorname{bjny} 1+\operatorname{cknz} 1+\operatorname{dlne} 1<\mathbf{C} \rightarrow(4.2) \\
\text { ai(3)+ bj(2)+ck(1)+dl(1)<c } \rightarrow(4.3)
\end{gathered}
$$

Then, the required bandwidth is assigned to each traffic class as follows 
The International Journal of Multimedia \& Its Applications (IJMA) Vol.3, No.2, May 2011

$$
\mathrm{a} 1(3)+\mathrm{b} 1(2)+\mathrm{c} 1(1)+\mathrm{d} 1(1)<\mathbf{C} \quad \rightarrow(4.4)
$$

The required bandwidth for voice calls i.e class-I traffic is always assigned the maximum bandwidth as cellular networks are primarily intended for voice services in addition to other services. If equation (4.4) is satisfied, then bandwidth upgradation is done starting from classII, then to class-III and class-IV in stepwise as follows.

$$
\mathrm{ai}(3)+\mathrm{bj}(2)+\mathrm{ck}(1)+\mathrm{dl}(1)<\mathbf{C} \quad \rightarrow(4.5)
$$

If equation (4.5) is satisfied, then bandwidth is upgraded in stepwise manner. This procedure is followed till the upgradation of $\mathrm{d} 2$ in equation (4.2) is satisfied.If equation (4.2) is not satisfied at any step, then bandwidth degradation is performed starting from class-IV, then class-III and finally for class II in stepwise manner till equation (4.2) is satisfied.

If a new call arrives in each class then the state space changes from $(3,2,1,1)$ to $(4,3,2,2)$ and the state space parameter for $(4,3,2,2)$ is $(\mathrm{nx} 2, \mathrm{ny} 2, \mathrm{nz} 2, \mathrm{ne} 2)$.

Let $\Delta \mathrm{nx} 1=\mathrm{nx} 2-\mathrm{nx} 1, \Delta \mathrm{nz1}=\mathrm{nz2}-\mathrm{nz} 1, \Delta$ ny1=ny2-ny1 and $\Delta$ ne1=ne2-ne1, where ' $\Delta$ 'represents the increase in the number of current calls when compared to the previous state of the calls.If the state space equation of $(3,2,1,1)$ is represented as $\mathrm{A}$, then the new state space equation for $(3,2,1,1)$ and $(4,3,2,2)$ becomes

$$
\begin{aligned}
& \mathrm{A}+\mathrm{a} 1 \Delta \mathrm{nx} 1+\mathrm{b} 1 \Delta \mathrm{ny} 1+\mathrm{c} 1 \Delta \mathrm{nz} 1+\mathrm{d} 1 \Delta \mathrm{ne} 1<\mathbf{C} \quad \rightarrow(4.6) \\
& \mathrm{A}+(\mathrm{a} 1 \Delta \mathrm{nx} 1+\mathrm{b} 1 \Delta \mathrm{ny} 1+\mathrm{c} 1 \Delta \mathrm{nz} 1+\mathrm{d} 1 \Delta \mathrm{ne} 1)<\mathbf{C} \quad \rightarrow(4.7) \\
& \text { \{ B }\}
\end{aligned}
$$

The necessary and sufficient condition for bandwidth adaptation is given in equation (4.8)

$$
(\mathrm{A})+(\mathrm{B})<\mathrm{C} \quad \rightarrow(4.8)
$$

If equation (3.7) is not violated, then the bandwidth Upgradation for the new state space is performed in stepwise as follows until equation (4.8) is satisfied.

$$
\begin{aligned}
& \mathrm{A}+(\mathrm{a} 1 \Delta \mathrm{nx} 1+\mathrm{b} 2 \Delta \mathrm{ny} 1+\mathrm{c} 1 \Delta \mathrm{nz} 1+\mathrm{d} 1 \Delta \mathrm{ne} 1)<\mathrm{C} \rightarrow(4.9) \\
& \mathrm{A}+(\mathrm{a} 1 \Delta \mathrm{nx} 1+\mathrm{b} 3 \Delta \mathrm{ny} 1+\mathrm{c} 1 \Delta \mathrm{nz} 1+\mathrm{d} 1 \Delta \mathrm{ne} 1)<\mathrm{C} \rightarrow(4.10) \\
& \mathrm{A}+(\mathrm{a} 1 \Delta \mathrm{nx} 1+\mathrm{b} 3 \Delta \mathrm{ny} 1+\mathrm{c} 2 \Delta \mathrm{nz} 1+\mathrm{d} 1 \Delta \mathrm{ne} 1)<\mathrm{C} \rightarrow(4.11) \\
& \mathrm{A}+(\mathrm{a} 1 \Delta \mathrm{nx} 1+\mathrm{b} 3 \Delta \mathrm{ny} 1+\mathrm{c} 2 \Delta \mathrm{nz} 1+\mathrm{d} 2 \Delta \mathrm{ne} 1)<\mathrm{C} \rightarrow(4.12)
\end{aligned}
$$

If at any stage of equation (4.8), (4.9), (4.10), (4.11), (4.12) and (4.13) is not valid, then bandwidth degradation is performed immediately to serve the requesting users. Bandwidth degradation is also performed in stepwise starting from low priority calls of class-IV, then in class-III and finally till it reaches b1 of class-II. The stepwise bandwidth degradation starts from equation (B) and then in equation (A) as to maintain the first come, first serve basis criteria. 
The International Journal of Multimedia \& Its Applications (IJMA) Vol.3, No.2, May 2011

Bandwidth adaptation algorithm for State space $(3,2,1,1)$ is done as follows

\begin{tabular}{|l|l|l|cc|l|}
\hline Traffic type & $\begin{array}{l}\text { Number of } \\
\text { calls }\end{array}$ & $\begin{array}{l}\text { Bandwidth } \\
\text { requirement in } \\
\text { Mbps }\end{array}$ & \multicolumn{2}{|c|}{$\begin{array}{l}\text { Bandwidth upgradation } \\
\text { Step1 Step 2 }\end{array}$} & $\begin{array}{l}\text { Bandwidth } \\
\text { degradation }\end{array}$ \\
\hline Class-I & 3 & 0.09 & 0.09 & 0.09 & ---- \\
\hline Class -II & 2 & 4 & 8 & 10 & --- \\
\hline Class-III & 1 & 1 & 2 & 2 & --- \\
\hline Class-IV & 1 & 0.003 & 0.02 & 0.02 & - \\
\hline $\begin{array}{l}\text { Total required } \\
\text { bandwidth in } \\
\text { Mbps }\end{array}$ & $5.093<\mathrm{C}$ & $9.093<\mathrm{C} \quad 12.11<\mathrm{C}$ & \\
\hline
\end{tabular}

State space $(4,3,2,2)$ i.e arrival of new call in each class is assigned bandwidth as follows

\begin{tabular}{|l|l|l|cc|l|}
\hline Traffic type & Number of & \multicolumn{2}{|l|}{$\begin{array}{l}\text { Bandwidth } \\
\text { calls }\end{array}$} & $\begin{array}{l}\text { Bandwidth upgradation } \\
\text { requirement in }\end{array}$ & \multicolumn{2}{|l|}{$\begin{array}{l}\text { Bandwidth } \\
\text { Step 1 Step 2 }\end{array}$} \\
\hline Class-I & 4 & 0.12 & 0.09 & 0.09 & ---- \\
\hline Class -II & 3 & & 8 & 10 & --- \\
\hline Class-III & 2 & & 2 & 2 & --- \\
\hline Class-IV & 2 & & 0.02 & 0.02 & \\
\hline $\begin{array}{l}\text { Total required } \\
\text { bandwidth in } \\
\text { Mbps }\end{array}$ & & & $9.093<\mathrm{C} \quad 12.11<\mathrm{C}$ & \\
\hline
\end{tabular}

Thus, by adapting the bandwidth requirement as when demand arises, more number of users could be served with acceptable level QoS. This sort of bandwidth adaptation also increases the effective utilization of network resources by serving more users when compared with conventional system. In the conventional system, the requested maximum bandwidth of each requesting traffic class is allotted to users based on the availability of the bandwidth. If more users demand for bandwidth, they are denied service abruptly due to the lack of bandwidth adaptation. But in the proposed method, bandwidth adaptation is carried over within the available bandwidth to serve more number of users thereby improving the network efficiency. [19] proposed channel sharing by assuming same traffic class in a cell, whereas the proposed framework with adaptive bandwidth allocation has simulated for four different traffic class and it is the modified scheme of [19].The detailed flow chart for bandwidth adaptation is given below 
The International Journal of Multimedia \& Its Applications (IJMA) Vol.3, No.2, May 2011

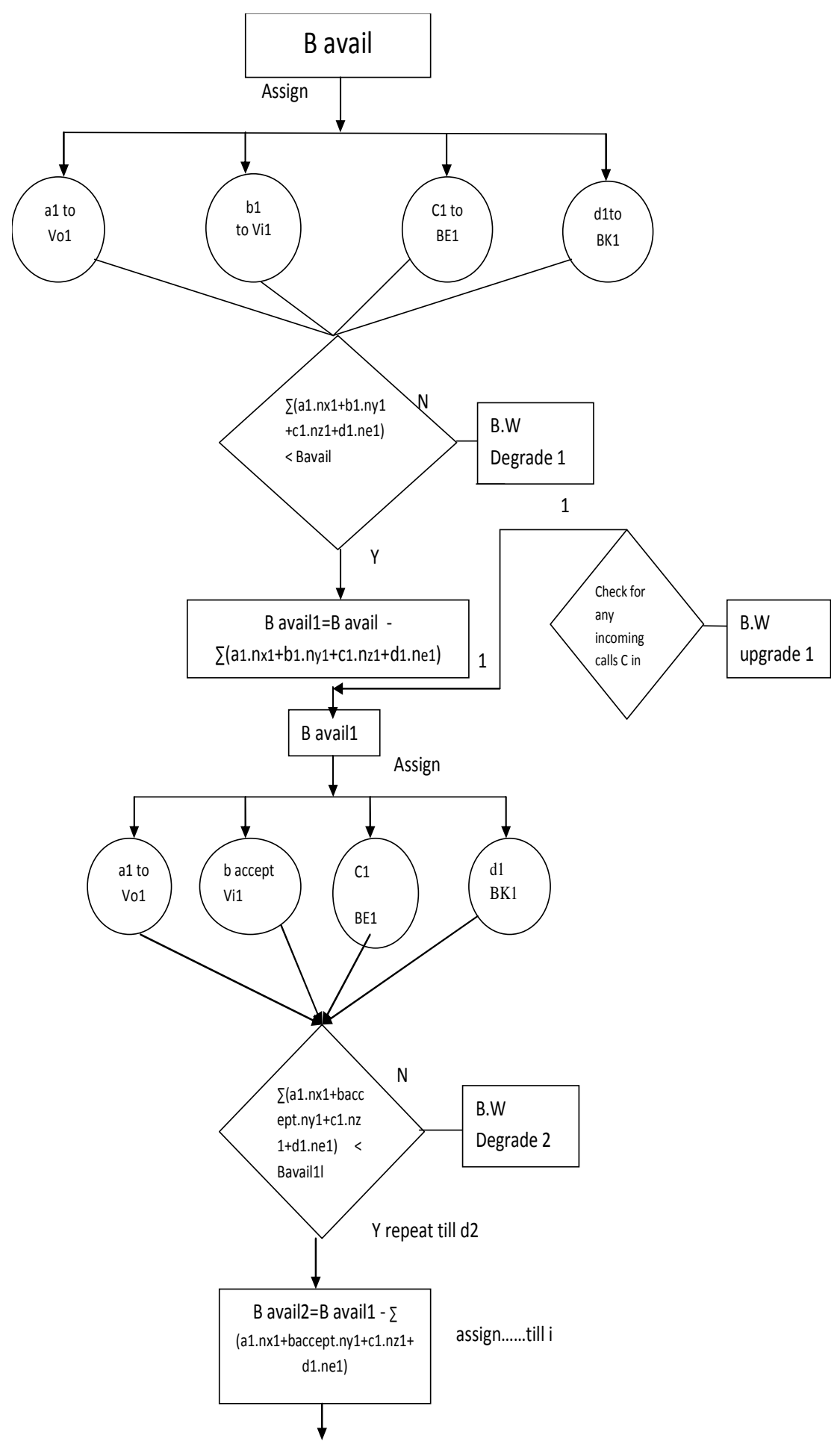

Flow chart of Bandwidth adaptation algorithm 
The International Journal of Multimedia \& Its Applications (IJMA) Vol.3, No.2, May 2011

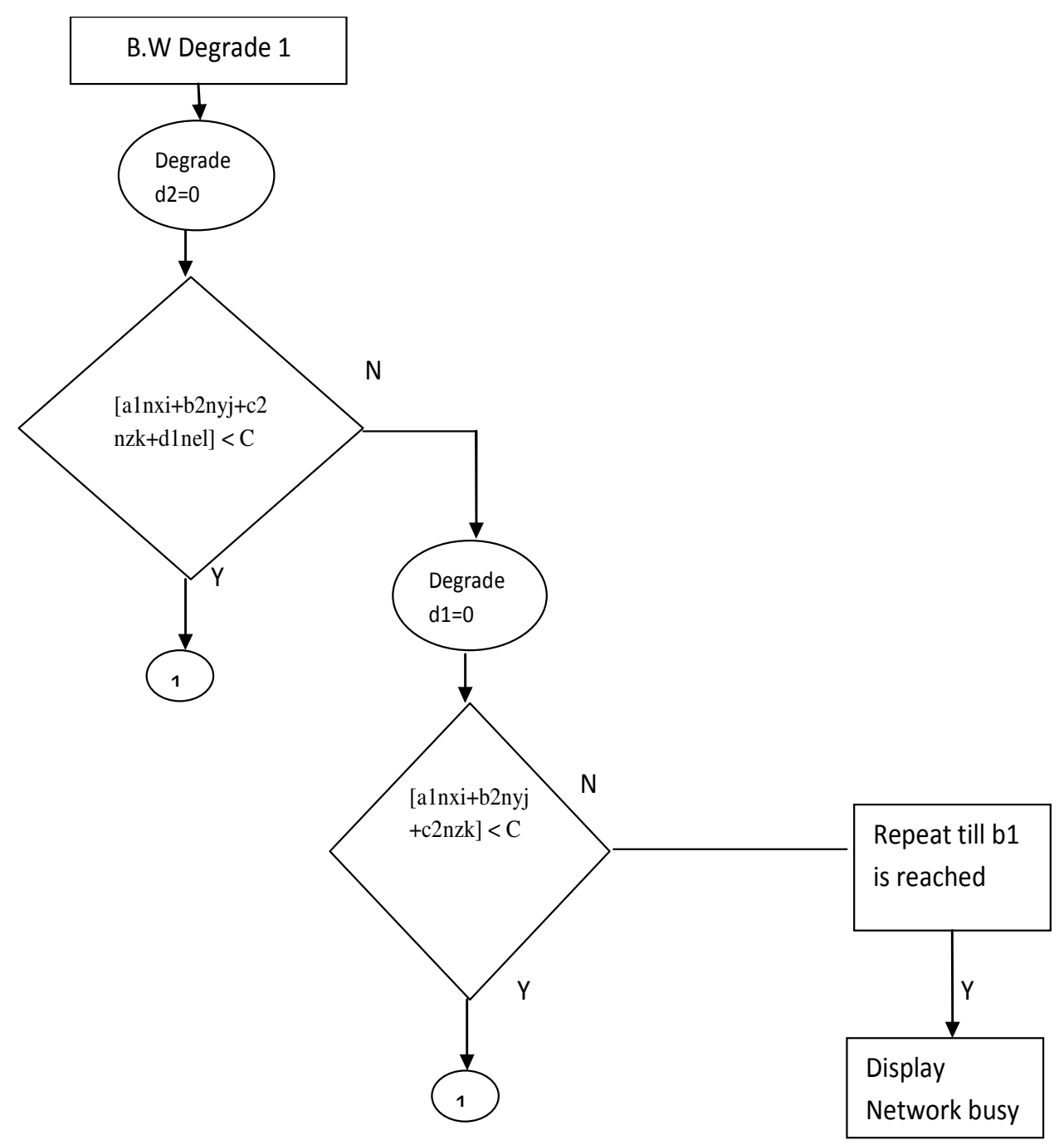

Flow Chart for Bandwidth Degradation

Flow chart of Bandwidth Degradation Process 
The International Journal of Multimedia \& Its Applications (IJMA) Vol.3, No.2, May 2011

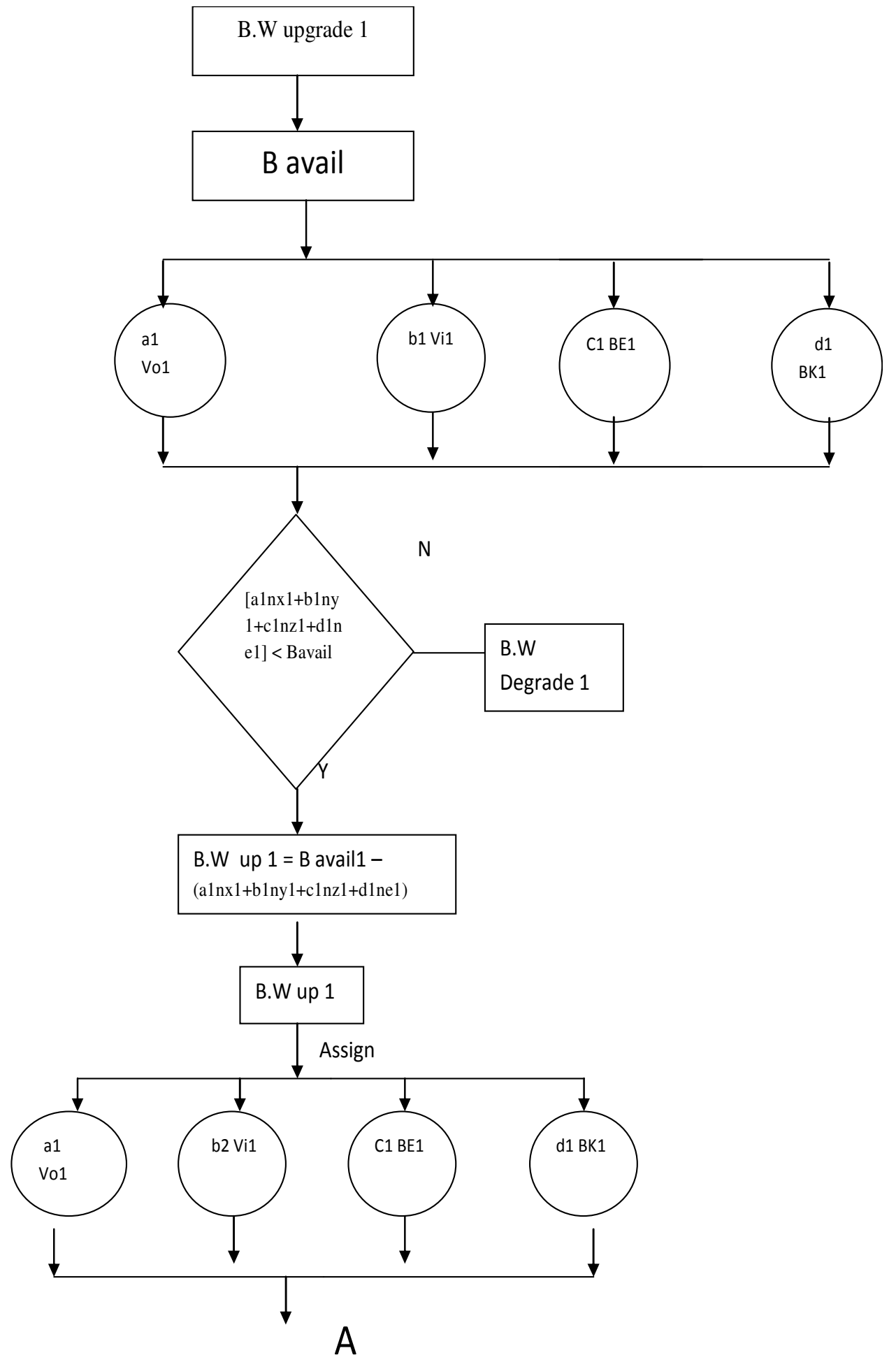


The International Journal of Multimedia \& Its Applications (IJMA) Vol.3, No.2, May 2011

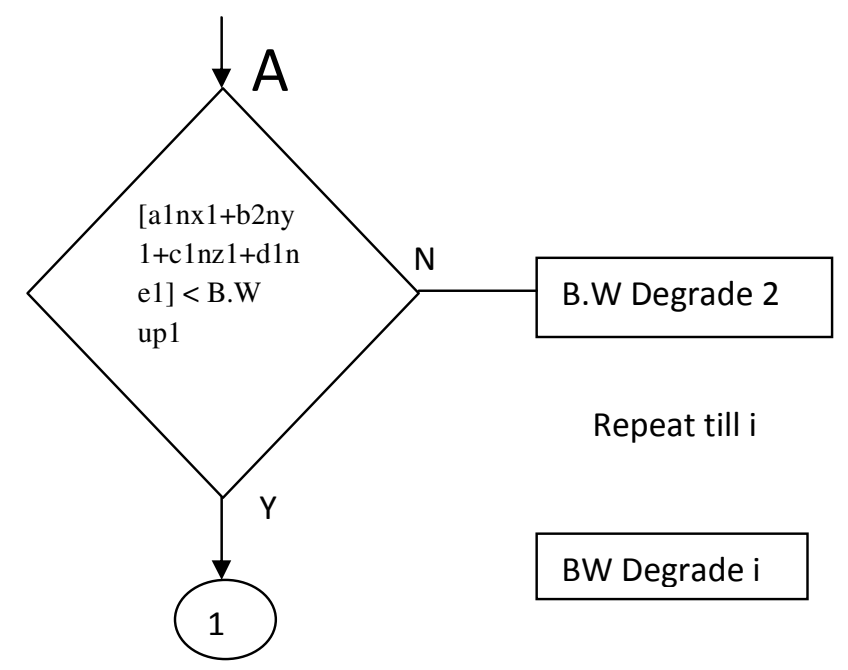

Flow chart for Bandwidth Upgradation Process

\subsection{Cross-Layer interaction among Application, MAC and Physical layer:}

Cross-Layer interaction is done among Application, MAC and Physical layers to adapt the available bandwidth so as to increase the overall network capacity with better Quality of Service (QoS) for multimedia users. In the proposed framework, to achieve such a cooperative communication between layers bandwidth adaptation using an evolutionary cross-layer approach among Application layer, MAC layer and Physical layer to provide the required QoE with QoS to the requesting end users has been proposed. As Physical layer is the most time variant entity in a wireless communication system and since the physical state information can be sent to any layer to improve the system efficiency any layer can adapt to the state of the channel. A good channel condition has SNR between 10-20dB, where as a bad channel has SNR between 0-10dB and hence a matrix table(2) is presented to adapt the layer with appropriate modulation and coding for enhanced data rate under the given channel condition of the user.MAC layer responds to the Application layer with the results from Physical layer which in turn triggers the probe generator of MSC to send the response. MSC responds to BSC to serve the requested MS.

\begin{tabular}{|c|c|l|c|c|}
\hline S.No & $\begin{array}{l}\text { Transmission } \\
\text { power in watts }\end{array}$ & $\begin{array}{l}\text { Transmission } \\
\text { mode, Coding } \\
\text { rate }\end{array}$ & $\begin{array}{l}\text { Proposed } \\
\text { data rate in } \\
\text { Mbps }\end{array}$ & $\begin{array}{l}\text { Measured } \\
\text { SNR in dB }\end{array}$ \\
\hline 1 & 1 & QPSK, 1/2 & 1 & $<4$ \\
\hline 2 & $1.3-1.5$ & QPSK, 2/3 & 2 & $4-7.5$ \\
\hline 3 & $1.75-2$ & QPSK,7/8 & 5.5 & $7.5-11$ \\
\hline 4 & $2.6-5.25$ & $\begin{array}{l}16 \text { QAM,/2/3 } \\
\text { 64 QAM,7/8 }\end{array}$ & 11 & $11-21.94$ \\
\hline
\end{tabular}

Table (2) Adaptable parameters in Physical layer

This sort of adaptive bandwidth allocation as per the traffic type of the requesting improves throughput with better usage of network resources. The reduced latency due to fast parameter exchanges between the layers by cross-layer approach minimizes the end to end delay. This 
bandwidth adaptation is reflected as control probe signals to BSC. Moreover, control probe request and acknowledge signal are too small and contains only three fields. Probe request sets the flag $=0$. If adaptive bandwidth allocation is possible, MSC responds with probe ACK signal by setting flag $=1$ else flag $=0$. Thus merely transmitting and receiving control packets, service could be provided to the requesting calls with satisfied QoS thereby improving QoE of the users.

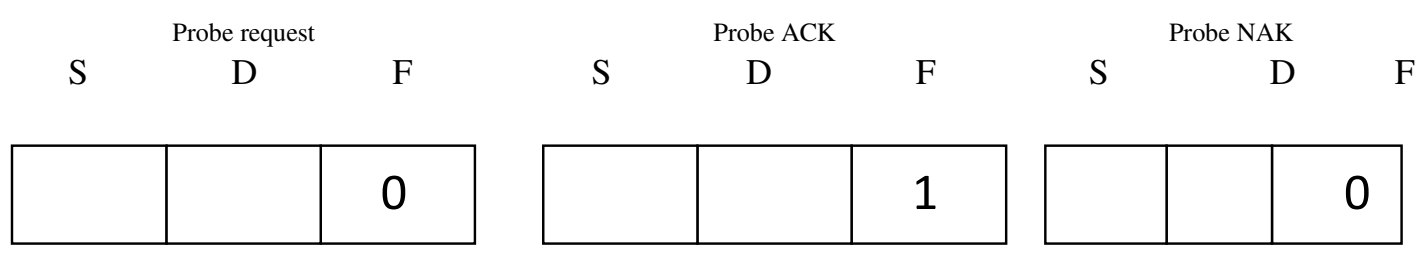

Frame format for Probe Signals

\section{RESULTS AND DISCUSSION}

The simulation is carried for 30 minutes for a hexagonal cell with the diameter of the cell as $1 \mathrm{Km}$ with a total capacity of $\mathrm{C}=16 \mathrm{Mbps}$. The average duration of each class is assumed for 3 minutes. The simulation is repeated for four different scenarios.

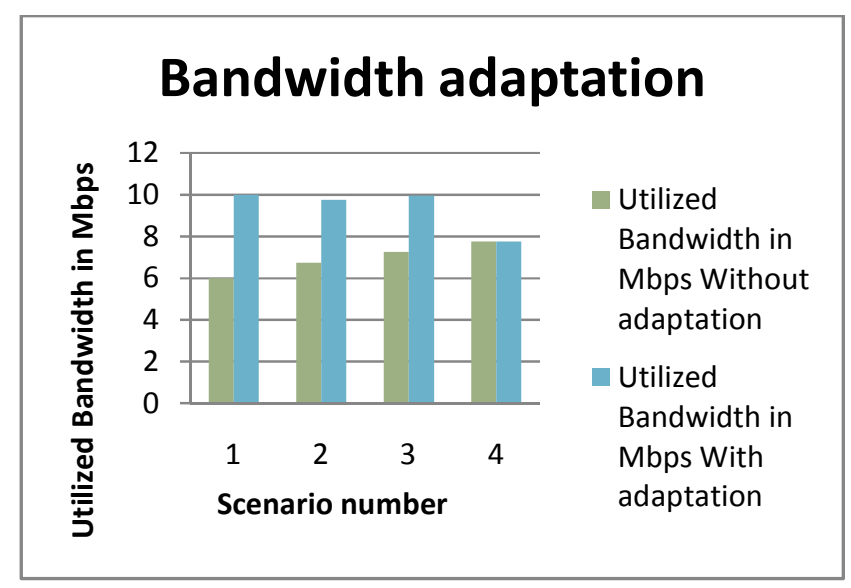

Figure 5.1 Utilized bandwidth versus adaptative traffic calls in terms of scenarios

From figure 5.1,it is very clear that the amount of bandwidth utilised under different scenarious comprising of majority of voice and video calls, video and best effort calls, video and background calls and video calls alone in different proportions is more when bandwidth adaptation is performed compared to the utilisation of bandwidth without bandwidth adaptation. This efficient bandwidth utilisation due to bandwidth adaptation using cross-layer concept based on traffic type and channel availability serves more number of multimedia users with QoE when compared with non-bandwidth adaptation. The increase in the utilised bandwidth by bandwidth adaptation ulitimately increases the efficiency of the system as shown in figure 5.2 and simulation results in table 5.3. 
The International Journal of Multimedia \& Its Applications (IJMA) Vol.3, No.2, May 2011

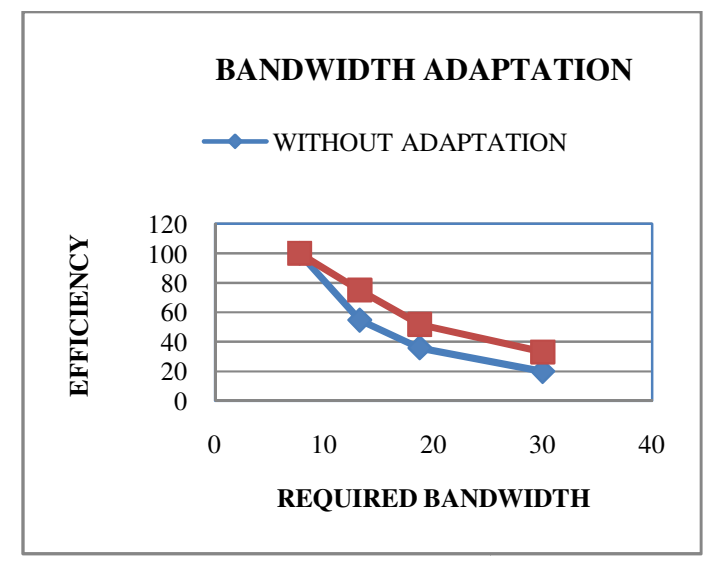

Figure5.2Requiredbandwidthversusefficiency

\begin{tabular}{|l|l|l|l|}
\hline Sno & $\begin{array}{l}\text { No. of } \\
\text { Nodes }\end{array}$ & $\begin{array}{l}\text { Service Delay } \\
\text { obtained from } \\
\text { non-bandwidth } \\
\text { adaptation } \\
\text { system(in } \\
\text { microseconds) }\end{array}$ & $\begin{array}{l}\text { Service Delay } \\
\text { obtained from } \\
\text { proposed } \\
\text { bandwidth } \\
\text { adaptation } \\
\text { system(in } \\
\text { microseconds) }\end{array}$ \\
\hline 1 & 4 & 2407.902 & 2402.902 \\
\hline 2 & 8 & 2407.602 & 2402.602 \\
\hline 3 & 12 & 2407.451 & 2402.451 \\
\hline 4 & 16 & 2407.451 & 2402.451 \\
\hline 5 & 20 & 2407.361 & 2402.361 \\
\hline & & & \\
\hline
\end{tabular}

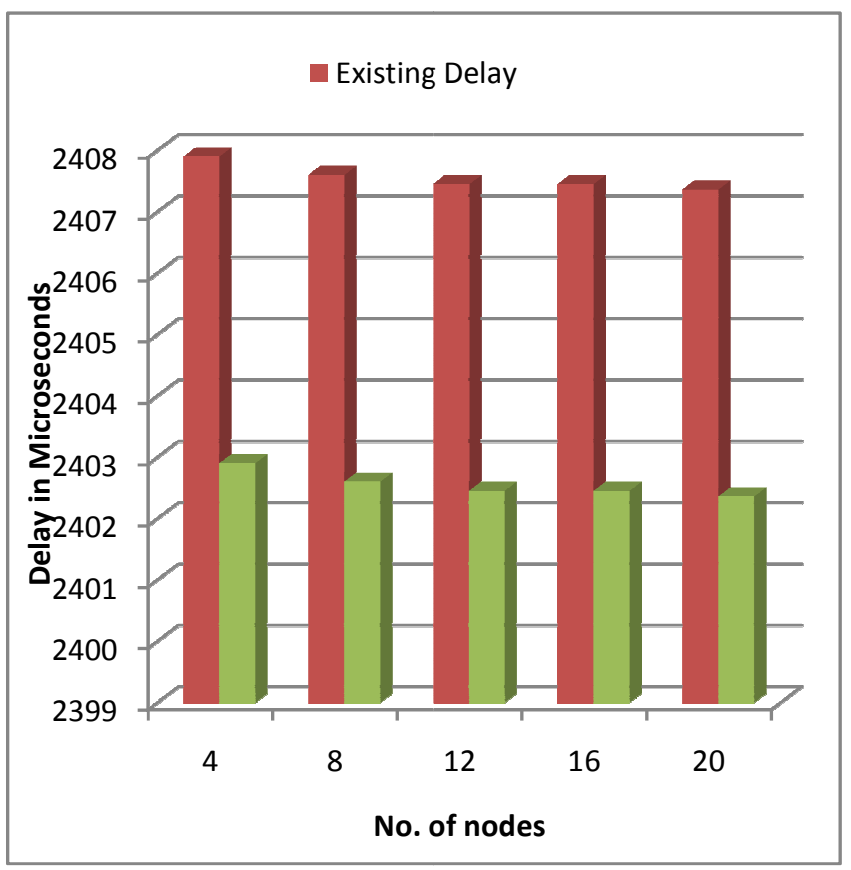

Figure 5.4: Comparison of service delay between non-bandwidth adaptation system and proposed bandwidth adaptative system .

The improvement in efficiency due to bandwidth adaptation reflects the improvised service to the requesting nodes and the simulation results shows that from table 5.3 and figure 5.4 ,a $5 \%$ improvement in service when compared to non bandwidth adaptation system. 
The International Journal of Multimedia \& Its Applications (IJMA) Vol.3, No.2, May 2011

The simulation results shows that due to better adaptation in bandwidth, modulation, coding, data rate and transmission power as per the channel condition of the requesting user, efficient usage of network resources with 16\% improvement in throughput and 5\% improvement in service provision of multimedia applications was achieved, thus providing QoS and QoE enhancement for multimedia users.

\section{CONCLUSION AND FUTURE WORK}

The proposed framework comprising of Cross-Layer Manager and Cross-Layer Coordinator along with adaptive bandwidth allocation improves the QoS of multimedia applications. Under scarce bandwidth conditions, the requesting users are served by dynamically varying the allocated bandwidth of the ongoing calls by bandwidth degradation process without compromising their acceptable level of QoS. However, maximum QoS is achieved during bandwidth up gradation, thereby satisfying the Quality of Experience (QoE) of end users. The framework was tested under various scenarios of heterogeneous traffic calls and the simulated results shows an average improvement of $16 \%$ in throughput and 5\% service rate when compared with non-adaptive system. However, the work could further be improved when crosslayer adaptation is implemented in network layer in addition to Physical, MAC and Application layer.

\section{REFERENCES}

[1] Hsiao-Hwa Chen,Jie Li,Yang Yang et al,"Challenges and Futuristic Perspective of CDMA Technologies:OCC-CDMA/OS for 4G Wireless Networks",IEEE 2006 proceedings,page no.39843989.

[2] Haui Paloheimo,Jukka Manner,Johanna Nieminen,Antti Yla- Jaaski,"Challenges in Packet Scheduling in 4G Wireless Networks", The $17^{\text {th }}$ Annual IEEE International Symposium on Personal,Indoor and

Mobile Radio Communications PIMRC'06.

[3] Hai Jiang,Weihua Zhuang and Xuenin,"Cross-Layer Design for Resource Allocation in a 3G Wireless

Networks and Beyond”,IEEE Communications Magazine,December 2005.

[4] Rosilah Hassan, James Irvine, Ian Gloves,'Implementations QoS onto virtual Bus Network" European Journal of Scientific Research, ISSN1450-216x, Vol25. No.1 (2009).

[5] SanjayShakkotai, Theodore S.Rappaport \& Peter C. Karlson, "Cross-Layer Design for Wireless Networks", IEEE Wireless Communication, June 2003, page 2-14.

[6] Viyay.T.Raisinghani \& Sridhar Iyer, "Cross-Layer Design Optimization in Wireless Protocol Stacks", Elsevier Science, March 2003.

[7] C.Verikoukis,L.Alonso and T.Giamalis,"Cross-Layer Optimization for Wireless Systems: A EuropeanResearch Key Challenge”,Global Communications Newsletter,July 2005.

[8] IEEE Vechicular Technology, Sep 06, Vol.1, No.3

[9] M.Rubaiyat Kibria \& Abbas Jamalipour, "On Designing Issues of the NGMN", IEEE Networks, Jan/Feb 2007, Vol21, No1 page 54-57. 
The International Journal of Multimedia \& Its Applications (IJMA) Vol.3, No.2, May 2011

[10] Bader Al-Manthari, Hossam Hassanein, Nidal Nasser, Schmid, "Packet Scheduling in 3.5G High Speed Downlink Packet Access Networks”, IEEE Network, Jan/Feb 07, Vol.21, No.1, pp 52-57.

[11] Tianmin Ren, Iordanis Koutsopoulos7Lean dros Tassiulus, "QOS Provisioning for Real Time traffic in Wireless Packet Networks”, IEEE GLOBECOM 2002.

[12] Zhikui Chen, “A Customizable QOS Strategy for Convergent Heterogeneous Wireless Communication”, IEEE Wireless Communication April 07, Vol.14, No.2.

[13] CorneliaKappler, Petteri poyhonen, MartinJohnsson, Stefan Schmid, "Dynamic Network Composition for beyond 3G Networks: A 3GPP viewpoint”, IEEE Network, Jan/Feb 07, Vol.21, No.1, pp 74-77.

[14] Chen Yiping, Yang Yuhang, "A New 4G Architecture Providing Multimode Terminals Always Best Connected Services”, IEEE Wireless Communication April 07, Vol.14, No.2, pp 33-39.

[15] Implementable Wireless Access for B3G Networks, IEEE Communication, March 07, Vol.45, No.3, Jongmin Jeong, Zygmunt J.Hass, "An Integrated Security Frame work for OWN Architecture", IEEE Wireless Communication, April 07, Vol.14,No.2.

[16] YoujunGao, Hui Tian, Lei Sun, Haibo Xu, "Research on the Access Network and MAC Technique Form Beyond 3G Systems”, IEEE Wireless Communication April 07, Vol.14, No.2, pp67-73.

[17] Yi Bing Lin,Ching-Feng Liang et al,'NTP-SIOT: A Test tool for Advanced Mobile Services, IEEE Networks,Jan/Feb 2007.

[18] Young-JuneChoi, KwangBok Lee, Saewoong Bahk, “All- IP 4G Network Architecture for Efficient Mobility and Resource Management”, IEEE Wireless Communication April 07, Vol.14, No.2, pp 76-81.

[19] Wei Li,Xiuli,"Call Admission Control for an Adaptive Heterogeneous Multimedia Mobile Network",IEEE Transactions on Wireless Communications,vol.6,NO.2,February 2007.

[20] Jiang Zhu and Abraham O.Fapojurvo,"A New Call Adnission Control method for providing desired throughput and delay performance in IEEE 802.11e Wireless LANs”,IEEE Transactions on Wireless Communication volume 6,No:2,February 2007.

[21] Gustavo Carnerio, Jose Ruela and Manuel Ricardo, "Cross-Layer Design in 4G Wireless Terminals", IEEE Wireless Communication, April 2004, page 7-13

[22] LodewukvanHoesel, Tim Nieberg, Jian Wu, Paul J.M.Havinga, "Prolonging the lifetime of wireless sensor networks by cross-layer interaction”, IEEE Wireless Communication, Dec 04, Vol.11. No.6, pp 61-65.

[23] Vikas- Kawadia \& P.R.Kumar, “A cautionary Perspective on cross-layer design”, IEEE Wireless Communications, Feb 05, Vol.12.No.1, pp 57-62. 
The International Journal of Multimedia \& Its Applications (IJMA) Vol.3, No.2, May 2011

\section{Authors}

M.Bhuvaneswari is Assistant Professor in the department of Electronics and Communication Engineering, Anna University of Technology, Tiruchirappalli, Tamilnadu, India. Her research interest includes Computer networks, Next generation networks and communication systems.

P.Seethalakshmi is the Director of Centre for Academic Excellence, Anna University of Technology, Tiruchirappalli,Tamilnadu, India. Her research interest includes Multimedia systems, Computer networks and Network Processors. 